\title{
(2) OPEN ACCESS \\ Effects of human immunodeficiency virus status on symptom severity in influenza-like illness in an otherwise healthy adult outpatient cohort
}

\author{
Rhonda E Colombo (1) , 1,2,3 Christina Schofield, ${ }^{1,2}$ Stephanie A Richard, ${ }^{2,3}$ \\ Mary Fairchok, 1,2,3 Wei-Ju Chen, ${ }_{1}^{2,3}$ Patrick J Danaher, ${ }_{1}^{4}$ Tahaniyat N Lalani, ${ }^{2,3,5}$ \\ Michelande Ridoré, ${ }^{2,3}$ Ryan C Maves, ${ }^{6}$ John C Arnold ${ }_{1}^{7}$ Anuradha Ganesan, ${ }^{2,3,8}$ \\ Brian Agan, ${ }^{2,3}$ Eugene V Millar, ${ }^{2,3}$ Christian Coles, ${ }^{2,3}$ Timothy H Burgess ${ }^{2}$
}

Additional supplemental material is published online only. To view, please visit the journal online (http://dx. doi.org/10.1136/jim-2020001694).

For numbered affiliations see end of article.

\section{Correspondence to} Dr Rhonda E Colombo and Dr Christina Schofield, Department of Medicine, Madigan Army Medical Center, Tacoma, WA 98431 5000, USA; rhonda.e.colombo.ctr@ mail.mil, christina.m.schofield2.civ@ mail.mil

Accepted 31 March 2021 Published Online First 23 April 2021

\section{Check for updates}

(C) American Federation for Medical Research 2021. Re-use permitted under CC BY-NC. No commercial re-use. Published by BMJ.

To cite: Colombo RE, Schofield C, Richard SA, et al. J Investig Med 2021;69:1230-1237.

\section{ABSTRACT}

The impact of HIV on influenza-like illness (ILI) has been incompletely described in the era of combination antiretroviral therapy, particularly in the post-H1N1 pandemic period. This analysis informs on ILI in an otherwise healthy, predominantly outpatient cohort of adults with HIV in the USA. From September 2010 to March 2015, this multisite observational cohort study enrolled otherwise healthy adults presenting to a participating US military medical center with ILI, a subset of whom were HIV positive. Demographics, clinical data, and self-reported symptom severity were ascertained, and enrollees completed a daily symptom diary for up to 10 days. 510 men were included in the analysis; 50 (9.8\%) were HIV positive. Subjects with HIV were older and less likely to be on active duty. Rhinovirus and influenza A were the most commonly identified pathogens. Moderate-severe diarrhea $(p<0.001)$ and fatigue $(p=0.01)$ were more frequently reported by HIV-positive men. HIV positivity was associated with higher gastrointestinal scores, but not other measures of ILI symptom severity, after controlling for age, race, military status, and influenza season. Few were hospitalized. HIV-positive subjects had more influenza $B(p=0.04)$ and were more likely to receive antivirals ( $32 \%$ vs $6 \%, p<0.01)$. Antiviral use was not significantly associated with symptom scores when accounting for potential confounders. In this predominantly outpatient cohort of adult men, HIV had minimal impact on ILI symptom severity. Despite similar illness severity, a higher percentage of subjects with HIV reported undergoing antiviral treatment for ILI, likely reflecting differences in prescribing practices. Trial registration number: NCT01021098.

\section{INTRODUCTION}

The effects of HIV infection on influenza-like illness (ILI) in the era of effective combination antiretroviral therapy (cART), particularly in the post-2009 H1N1 influenza pandemic era, have not been well established. Available results vary, depending on the population studied and

\section{Significance of this study}

What is already known about this subject?

- The relative contribution of specific respiratory viruses to acute respiratory illness is likely to vary by season and patient population.

- HIV has been described as a risk factor for both acquisition and severity of acute respiratory infections in low-income and middle-income countries.

- The impact of HIV status on influenza and influenza-like illness (ILI) in the USA and other high-income countries is less clear particularly in the post-H1N1 pandemic period.

What are the new findings?

- In this predominantly outpatient cohort of men presenting with ILI, HIV positivity was independently associated with a higher composite gastrointestinal score, but not other measures of ILI symptom severity, after controlling for age, race, military status, and influenza season.

- Rhinovirus was the most common pathogen detected in both HIV-positive and HIV-negative subjects, followed by influenza $A$ virus.

- Influenza B was more frequently identified in subjects with HIV $(p=0.04)$; however, the absolute number of influenza $B$ cases was small.

- Despite similar illness severity and excellent HIV control, a higher percentage of subjects with HIV reported undergoing antiviral treatment for ILI, suggesting that prescribing practices favor more cautious treatment of ILI in individuals with HIV.

methodology used, thus limiting the applicability to the broader population.

In Africa, several studies have been performed suggesting that HIV is a risk factor for both acquisition and severity of acute respiratory 
Significance of this study

How might these results change the focus of research or clinical practice?

- These results may help to better inform the clinical risk assessment and management decisions for a man with well-controlled HIV who presents with an ILI.

infections (ARIs). A recent study from Malawi showed that the risk of influenza was $>2.5$-fold higher among HIVinfected adults as compared with HIV-uninfected adults after adjusting for demographic and socioeconomic factors. ${ }^{1}$ HIV was also independently associated with a nearly fivefold increased risk of severe disease (OR 4.98, 95\% CI 2.09 to 11.88$).{ }^{1} \mathrm{~A}$ meta-analysis of case fatality rates among 36 studies of ARI in Africa found an increased risk of death in HIV-infected as compared with HIV-uninfected (pooled OR $4.10,95 \%$ CI 2.63 to 6.27 ), with the highest risk of death occurring among HIV-infected children under the age of 5 years. ${ }^{2}$

The impact of HIV status on influenza and ILI in the USA and other high-income countries (HICs) is less clear. US studies performed earlier in the HIV epidemic identified HIV as a risk factor for increased influenza severity ${ }^{3}$; however, whether this association persists in the era of cART has been questioned. In a 2011 review, Sheth et al suggested that HIV infection does not appear to increase susceptibility to influenza in the USA, although available data were limited regarding the 2009 pandemic strain H1N1pdm09. ${ }^{5}$ Serological studies focusing on influenza incidence in the USA and Australia during the H1N1 pandemic did not detect any evidence of increased risk of influenza acquisition among HIV-infected individuals. ${ }^{6} \mathrm{~A}$ 2010-2011 multicenter prospective US cohort study found that the duration of influenza virus shedding among HIVinfected adults diagnosed with influenza, 95\% of whom were on cART, was similar to durations previously reported for HIV-uninfected individuals with influenza. ${ }^{7}$ Specifically with regard to pandemic influenza, several studies from HIC reported disproportionate rates of HIV among individuals hospitalized with H1N1pdm09 influenza infection as compared with the prevalence of HIV in the general population. ${ }^{8}$ However, in a 2009 study of hospitalized patients in the USA with H1N1pdm09, clinical outcomes, including rates of intensive care unit (ICU) admission, mechanical ventilation, and death, did not differ by HIV status. ${ }^{9}$ A similar study of patients hospitalized with influenza in Spain in 2009 also reported similar clinical outcomes between HIV-uninfected and HIV-infected individuals. ${ }^{10}$ However, in a separate Spanish study of patients with confirmed H1N1 admitted to the ICU during the 2009 pandemic and in the early postpandemic period (2010-2011), HIV was independently associated with a significantly increased ICU mortality in the postpandemic period (OR 19.835, 95\% CI 2.236 to 175.954 ) but not during the pandemic (OR 1.362 , $95 \%$ CI 0.279 to 6.648$).{ }^{11}$

There is a dearth of published information regarding the impact of HIV on influenza and ILI in the extended postpandemic period. A systematic review commissioned by the WHO to assess risk factors associated with severe outcomes from influenza in HIC versus low-income and middle-income countries (LMICs) attempted to assess the impact of HIV/AIDS, including data through March 2016. ${ }^{12}$ However, only approximately $10 \%$ of included studies collected postpandemic data, and few studies specifically addressed HIV as a separate comorbidity. For those that did, HIV/AIDS was associated with increased risk of ICU admission and/or death in LMIC but not in HIC. ${ }^{12}$ To our knowledge, there has been no analysis of ILI severity and outcomes in the postpandemic period among a predominantly outpatient cohort of otherwise healthy adults living with HIV in an HIC. Thus, the purpose of this analysis was to compare the prevalence and severity of symptoms as well as clinical outcomes associated with ILI in a US cohort of adult men with well-controlled HIV with otherwise healthy adult men who were HIV negative.

\section{METHODS}

\section{Overview of the Acute Respiratory Infection Consortium (ARIC) study}

Established in July 2009, the ARIC is a multisite, multidisciplinary clinical research network for the study of ILI in US Department of Defense healthcare facilities. At the core of the ARIC is the Natural History Study, an observational, longitudinal cohort study to determine the etiology, epidemiology, and clinical characteristics of ILI among otherwise healthy military personnel and beneficiaries. ${ }^{13}$ Five military treatment facilities across the continental USA are participating: (1) Naval Medical Center Portsmouth, Virginia; (2) Naval Medical Center, San Diego, California; (3) Madigan Army Medical Center, Tacoma, Washington; (4) Brooke Army Medical Center, San Antonio, Texas; and (5) Walter Reed National Military Medical Center, Bethesda, Maryland.

\section{Patient population and procedures}

Between September 2010 and March 2015, 1457 patients aged 0-65 years and presenting within 72 hours after onset of ILI, defined as having a temperature of $\geq 100.4^{\circ} \mathrm{F}$ and sore throat or one of the following respiratory symptoms: cough, sputum production, shortness of breath, or chest pain, were recruited. Both inpatient and outpatient subjects were eligible for participation. Subjects with a serious comorbidity other than HIV (diabetes, active malignancies, chronic obstructive pulmonary disease, severe asthma, a medical condition requiring immune modulating agents for $>5$ days within the last month, chronic neuromuscular disease, chronic kidney disease, or chronic heart disease), as well as individuals under the age of 18 years, were excluded from this analysis. Additionally, because the vast majority (95\%) of HIV-positive enrollees were male and sex has previously been associated with ILI severity in our cohort, ${ }^{14}$ we limited our analysis to male subjects. For the 15 individuals (10 HIV-negative and $5 \mathrm{HIV}$-positive) who experienced multiple ILIs, only the first ILI was retained for analysis.

At enrollment, demographic information, clinical symptoms, and vital signs were recorded by clinical research personnel using a standard questionnaire, and a nasopharyngeal (NP) swab (Nylon-flocked; Copan Diagnostics, Corona, California, USA) for respiratory viral detection was collected. After enrollment (day 0), subjects returned 
Table 1 Demographic characteristics of adult, male service members and DOD beneficiaries presenting with ILI, by HIV status

\begin{tabular}{|c|c|c|c|}
\hline & HIV+ & HIV- & \multirow[b]{2}{*}{$P$ value } \\
\hline & $\mathrm{n}=50(\%)$ & $\mathrm{n}=460(\%)$ & \\
\hline \multicolumn{4}{|l|}{ Age (years) } \\
\hline $18-24$ & $6(12)$ & $126(27)$ & $<0.001$ \\
\hline $25-34$ & $24(48)$ & $210(46)$ & \\
\hline $35-44$ & $6(12)$ & $94(20)$ & \\
\hline $45+$ & $14(28)$ & $30(7)$ & \\
\hline \multicolumn{4}{|l|}{ Race } \\
\hline White & $21(42)$ & $247(54)$ & 0.3 \\
\hline Black & $9(18)$ & $51(11)$ & \\
\hline Hispanic & $12(24)$ & $103(22)$ & \\
\hline Unknown/other & $8(16)$ & $59(13)$ & \\
\hline \multicolumn{4}{|l|}{ Military status } \\
\hline Active duty & $37(74)$ & $429(93)$ & $<0.001$ \\
\hline Retired & $11(22)$ & $17(4)$ & \\
\hline Dependent & $2(4)$ & $14(3)$ & \\
\hline \multicolumn{4}{|l|}{ Education } \\
\hline$>$ High school & $20(40)$ & $202(44)$ & 0.69 \\
\hline High school & $30(60)$ & $257(56)$ & \\
\hline Missing & $0(0)$ & $1(0)$ & \\
\hline \multicolumn{4}{|l|}{ Season } \\
\hline $2010 / 11$ & $13(26)$ & $157(34)$ & 0.21 \\
\hline $2011 / 12$ & $15(30)$ & $128(28)$ & \\
\hline $2012 / 13$ & $11(22)$ & $58(13)$ & \\
\hline 2013/14 & $6(12)$ & $87(19)$ & \\
\hline 2014/15 & $5(10)$ & $30(7)$ & \\
\hline \multicolumn{4}{|l|}{ Time since influenza vaccination (days) } \\
\hline $14-59$ & $8(16)$ & $69(15)$ & 0.93 \\
\hline $60-89$ & $6(12)$ & $55(12)$ & \\
\hline 90-119 & $7(14)$ & $77(17)$ & \\
\hline 120-149 & $6(12)$ & $70(15)$ & \\
\hline $150+$ & $23(46)$ & $180(39)$ & \\
\hline Missing & $0(0)$ & $9(2)$ & \\
\hline $\begin{array}{l}\text { Time from ILI onset to seeking healthcare } \\
\text { (days) }\end{array}$ & $2(0,3)$ & $2(0,6)$ & 0.7 \\
\hline \multicolumn{4}{|l|}{ Smoking status } \\
\hline Current & $10(20)$ & $101(22)$ & 0.13 \\
\hline Former & $16(32)$ & 89 (19) & \\
\hline Non-smoker & $21(42)$ & $253(55)$ & \\
\hline Missing/N/A & $3(6)$ & $17(4)$ & \\
\hline
\end{tabular}

$P$ values calculated using $\chi^{2}$ or Fisher's exact test. Statistically significant at a $p$ value of $<0.05$ (in bold).

DoD, Department of Defense; ILI, influenza-like illness; N/A, not applicable.

for visits at three subsequent time points: days $3 \pm 1$, $7 \pm 2$ and $28 \pm 7$. Clinical symptoms and vital signs were recorded, and an NP swab was collected at each visit; if a person tested positive for a pathogen on any of the collected swabs, they were considered positive for that pathogen. The date of the most recent influenza vaccination was reported by the subject. Written informed consent was obtained at enrollment.

\section{Detection of respiratory viruses}

In addition to standard of care clinical testing performed at the military treatment facility (eg, rapid antigen testing for influenza A, influenza $\mathrm{B}$, and respiratory syncytial virus (RSV)), each subject consented to collection of an NP swab

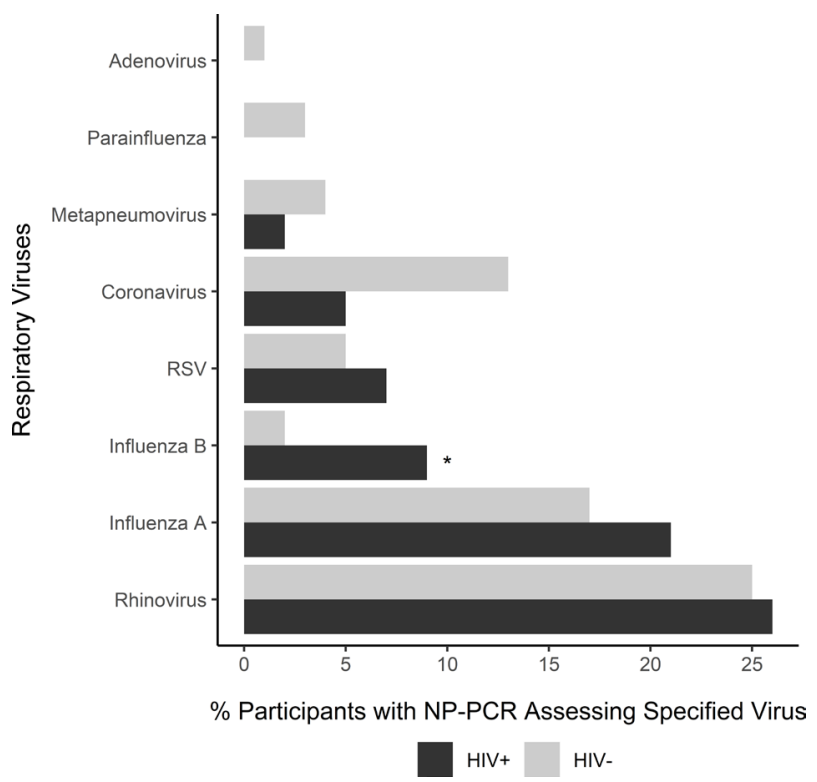

Figure 1 Distribution of respiratory viruses isolated by RT-PCR from NP swabs among subjects with ILI, categorized according to HIV positivity. The bars represent the percentage of participants with a detected respiratory virus among those who had a documented NP PCR specimen result for any of the specified viruses. Multiple viruses may have been identified from a single sample. The number of sample results varied by virus (adenovirus, $n=502$; parainfluenza virus, $n=469$; metapneumovirus, $n=469$; $\mathrm{RSV}, \mathrm{n}=469$; coronavirus, $\mathrm{n}=425$; influenza, $\mathrm{n}=503$; and rhinovirus, $\mathrm{n}=489$ ). * $S$ tatistically significant at a $p$ value of $<0.05$, Fisher's exact test. ILI, influenza-like illness; NP, nasopharyngeal; RSV, respiratory syncytial virus; $R T-P C R$, reverse transcription-PCR.

for further viral diagnostics. On collection, the NP swab was placed immediately into viral transport media, frozen at $-80^{\circ} \mathrm{F}$, and shipped on dry ice to the Naval Health Research Center (San Diego, California, USA). Molecular diagnostics, including singleplex and multiplex assays, were used to test specimens for common respiratory viruses (eg, human rhinovirus, influenza A, influenza B, adenovirus, RSV, coronavirus, parainfluenza virus, and human metapneumovirus).

\section{Clinical characteristics and severity measures}

The presence of clinical symptoms and the severity of each symptom were recorded by self-report as 0 , none; 1 , mild; 2 , moderate; and 3, severe. Subjects were trained by clinical research personnel on the definitions of each score. Symptom severity was quantified using the following six measures: (1) individual symptom score for 20 symptoms; (2) upper respiratory symptom score, calculated as the sum of severity scores for earache, runny nose, sore throat and sneezing; (3) lower respiratory symptom score, calculated as the sum of severity scores for cough, difficulty breathing, hoarseness and chest discomfort; (4) composite systemic symptom score, calculated as the sum of severity scores for chills, muscle ache, headache, and fatigue; (5) gastrointestinal (GI) score, calculated as the sum of severity scores for nausea, vomiting, diarrhea, abdominal pain, and anorexia; and (6) total composite score, calculated as the sum of 
Table 2 Symptoms reported by study subjects experiencing ILI by HIV status

\begin{tabular}{|c|c|c|c|}
\hline & HIV+ & HIV- & $P$ value \\
\hline Moderate-severe symptoms & $\mathrm{n}=50(\%)$ & $n=460(\%)$ & \\
\hline Fatigue & $49(98)$ & $387(84)$ & 0.01 \\
\hline Muscle pain & $38(76)$ & $316(69)$ & 0.37 \\
\hline Cough & $38(76)$ & $371(81)$ & 0.55 \\
\hline Chills & $37(74)$ & $371(81)$ & 0.35 \\
\hline Reduced appetite & $36(72)$ & $295(64)$ & 0.34 \\
\hline Sore throat & $35(70)$ & $334(73)$ & 0.82 \\
\hline Headache & $32(64)$ & $318(69)$ & 0.56 \\
\hline Joint pain & $30(60)$ & $297(65)$ & 0.63 \\
\hline Hoarseness & $30(60)$ & $250(54)$ & 0.54 \\
\hline Runny nose & $30(60)$ & 317 (69) & 0.26 \\
\hline Diarrhea & $25(50)$ & $96(21)$ & $<0.001$ \\
\hline Sneezing & $21(42)$ & 205 (45) & 0.84 \\
\hline Itchy eyes & $18(36)$ & $150(33)$ & 0.74 \\
\hline Dizziness & $19(38)$ & $139(30)$ & 0.33 \\
\hline Shortness of breath & $18(36)$ & $158(34)$ & 0.94 \\
\hline Earache & $14(28)$ & $153(33)$ & 0.55 \\
\hline Nausea & $15(30)$ & $137(30)$ & 1.00 \\
\hline Abdominal pain & $12(24)$ & $104(23)$ & 0.96 \\
\hline Chest pain & $8(16)$ & $134(29)$ & 0.07 \\
\hline Vomiting & $6(12)$ & 67 (15) & 0.78 \\
\hline
\end{tabular}

Individual symptoms were recorded as none, mild, moderate or severe, generally for $7+$ days post-ILI onset ( $90 \%$ of subjects recorded at least 7 days with symptom data). The values reported here are the number (\%) of individuals who reported moderate or severe symptoms at any time during the episode. $\chi^{2}$ and t-test were used to calculate $p$ values. $P$ values were considered statistically significant at $<0.05$ (in bold).

ILI, influenza-like illness.

severity scores for upper respiratory, lower respiratory, systemic, and GI composite scores. Symptom data were collected for each day of the ILI; the maximum score for each symptom during the ILI was identified and used to calculate the episode composite scores. Hospitalization was recorded prospectively for the duration of the study.

\section{Statistical analysis}

We compared categorical variables (demographic and clinical characteristics, moderate-severe symptoms, isolated viral pathogens) by HIV status using $\chi^{2}$ tests or Fisher's exact test as appropriate. To assess differences between the groups for the symptom scores, we performed t-tests. $P$ values of $<0.05$ were considered statistically significant. Composite symptom scores during the episode were compared by HIV status using multivariate linear models, controlling for other factors such as influenza season, age, race, and military status. In addition, daily symptom scores were compared by HIV status using multivariate models with random effects for subjects, controlling for season and age. HIV-related enrollment and convalescent CD4 count, percent $\mathrm{CD} 4$, and viral load were compared using t-tests, and were presented in a figure with locally weighted scatterplot smoothing lines, that is, a running average. Viral load and CD4 count were normalized using log transformation prior to testing. Analyses were performed using R V.3.5.2, ${ }^{15}$ specifically the nlme package for the mixed effects models. ${ }^{16}$

\section{RESULTS}

\section{Patient demographics}

A total of 946 adult patients with ILI were enrolled in the ARIC Natural History Study between September 2010 and March 2015. Among them, 510 (54\%) were male and reported data on symptom severity and were thus eligible for inclusion in this analysis. Of these, 50 (9.8\%) were HIV positive without other significant comorbidities. Subjects infected with HIV were more likely to be older (mean age in years: HIV-positive $35.7 \pm 10.7$, HIV-negative $30.5 \pm 8.4$ ) and not on active military duty (26\% HIV-positive vs $7 \%$ HIV-negative). There were no differences noted in race, ethnicity, education level, smoking status, or time since seasonal influenza vaccination between HIV-positive and HIV-negative enrollees (table 1).

\section{Viral etiology}

Rhinovirus (25\%), influenza A (17\%), and coronavirus (12\%) were the most frequently identified viral pathogens (figure 1); no etiological agent was identified in $32 \%$ of the samples. In both HIV-positive and HIV-negative subjects, the majority $(71 \%$ and $89 \%$ of influenza cases, respectively) of confirmed influenza cases were due to influenza A (48.3\% H3N2, 41.4\% pdm09H1N1, 10.3\% not subtyped). The only detected difference in viral etiology by HIV status was that influenza $\mathrm{B}$ was more frequently identified in subjects with HIV $(\mathrm{p}=0.04)$, although the numbers were small $(\mathrm{n}=4(9 \%)$ in HIV-positive, $\mathrm{n}=10(2 \%)$ in HIVnegative). There were no definite trends in the timing of influenza B infections; although the highest number of influenza B cases were detected in December, infections occurred as early as September and as late as May.

\section{Clinical severity}

In terms of maximum individual symptom severity, there were few significant differences detected between HIVpositive and HIV-negative enrollees on unadjusted analysis (table 2).

HIV-positive enrollees were more likely to report moderate-severe diarrhea $(50 \%$ vs $21 \%, \mathrm{p}<0.001)$ and fatigue $(98 \%$ vs $84 \%, \mathrm{p}=0.01)$. However, the mean composite symptom scores (lower respiratory, upper respiratory, systemic, GI, and total) were similar between HIV-negative and HIV-positive enrollees. The daily trend in composite symptom scores was also similar irrespective of HIV status, with the exception that HIV-negative subjects had significantly higher upper respiratory scores on day 4, whereas HIVpositive subjects had higher GI scores on days 1, 2, and 5 (figure 2). On multivariate analysis, HIV infection was independently associated with a higher composite GI score, controlling for age, race, military status, and influenza season $(\mathrm{p}<0.05)$. Subjects who were in the age strata of 18-24 years also reported statistically higher composite GI scores than each of the older age groups, controlling for race, influenza season, HIV and military status (online supplemental table 1).

Regarding clinical outcomes, there was a similar rate of hospitalization ( $6 \%$ vs $4 \%, \mathrm{p}=0.45)$, pneumonia $(2 \%$ vs $1 \%, \mathrm{p}=0.46)$, and supplemental oxygen use $(0 \%$ vs $1 \%, \mathrm{p}=1.0$ ) between HIV-positive and HIV-negative 

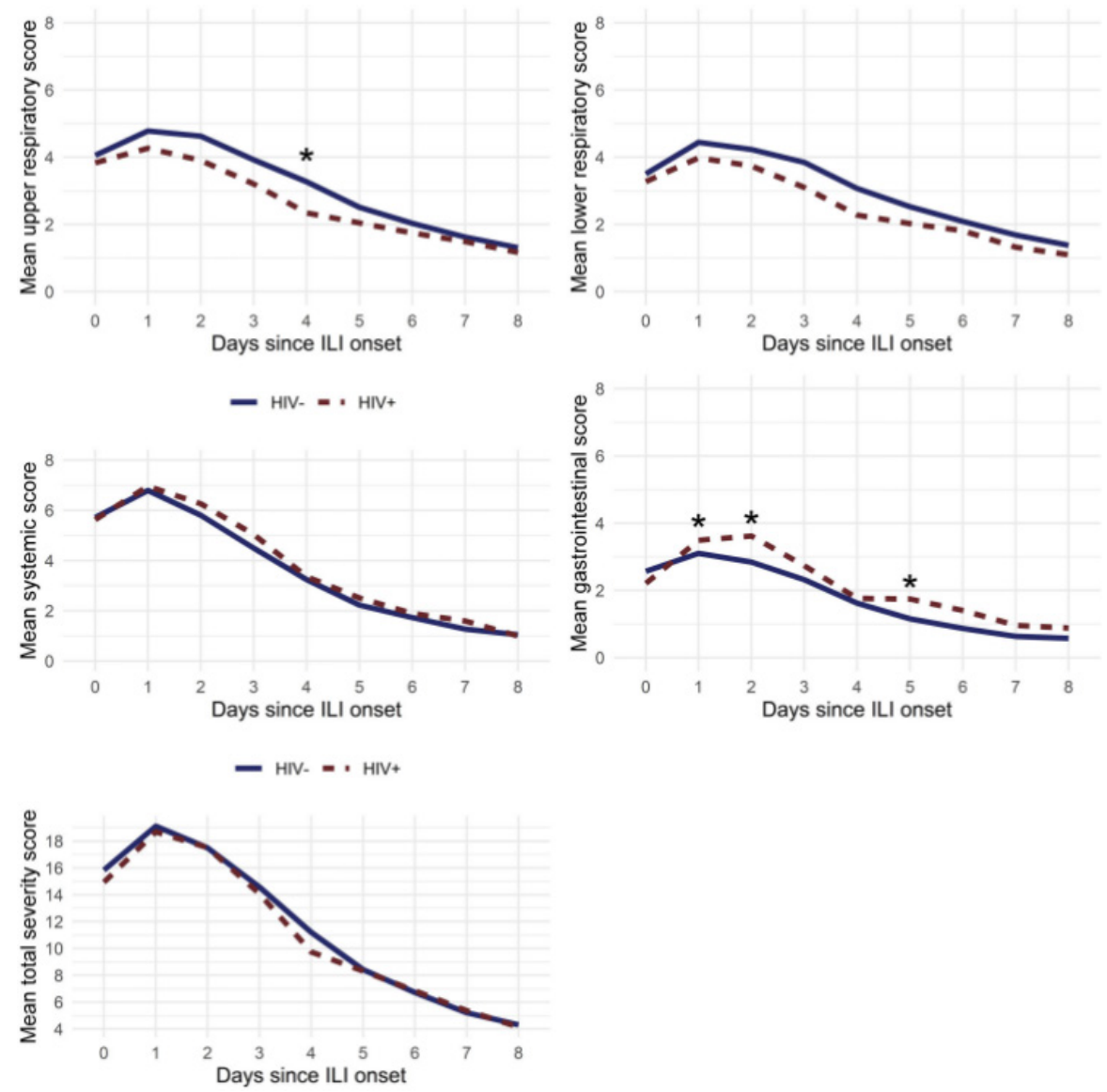

Figure 2 Trend in mean composite symptom score by day according to HIV status. *Statistically significant at a p value of $<0.05$ based on multivariate models that included an interaction between HIV status and day, as well as season and age, and a random effect for subject. ILI, influenza-like illness.

enrollees. Only one study subject required intensive care admission, and that person was HIV negative $(\mathrm{p}=1.00)$. HIV-positive and HIV-negative enrollees were equally likely to receive antibiotics $(22 \%$ vs $16 \%$, $\mathrm{p}=0.41$ ); however, HIV-positive enrollees reported taking antivirals directed at influenza more commonly than HIV-negative enrollees $(32 \%$ vs $6 \%, \mathrm{p}<0.01)$. When antiviral use was incorporated into the multivariate model, however, receipt of antivirals did not change the impact of HIV on any of the composite symptom scores, nor were antivirals independently associated with a difference in any of the symptom scores.

\section{HIV-related parameters during ILI}

HIV-related parameters from enrollment and convalescence were available for $42(84 \%)$ of the HIV-positive men. There was a significantly lower $(\mathrm{p}<0.001)$ absolute CD4 count at enrollment (median day 2 of illness) compared with convalescence (median day 30 of illness) (median 492 (IQR 364-634) vs 600 (IQR 503-688) cells $/ \mathrm{mm}^{3}$ ); however, there was no significant difference in \%CD4 at the two time periods (figure 3). The median HIV viral load was lower at the convalescent as compared with the enrollment visit (32 copies/mL (IQR 20-48) vs 48 copies/mL (IQR 20-60), p=0.01).

\section{DISCUSSION}

Respiratory viruses cause the majority of ARI in both children and adults. In this prospective study of otherwise healthy adult male US service members and beneficiaries who presented with ILI between 2010 and 2015, the most common viruses identified were rhinovirus, influenza $\mathrm{A}$, and coronavirus; the frequency of these viruses did not differ by HIV status. A significantly higher percentage of subjects with HIV had influenza $B$ identified than HIV-negative subjects; however, the total number of influenza B cases was small (14 overall: 4 in HIV-positive, 10 in HIV-negative). Thus, the clinical significance of this finding remains unclear. The relative contribution of specific respiratory viruses to acute respiratory illness is likely to vary by season and patient population. For instance, in a Canadian study of 50 patients with well-controlled HIV (median CD4 325 cells $/ \mu \mathrm{L}$, median viral load $<50$ copies $/ \mathrm{mL}$ ) who presented with febrile respiratory illness during a 3-year time period (2003-2006), a respiratory virus was identified as a contributory pathogen in $64 \%$ of patients; influenza $\mathrm{A}$ and influenza B were equally prevalent and accounted for $65 \%$ of the viruses identified, followed by human metapneumovirus, RSV, and human coronavirus. ${ }^{17}$ Interestingly, although rhinovirus was included 

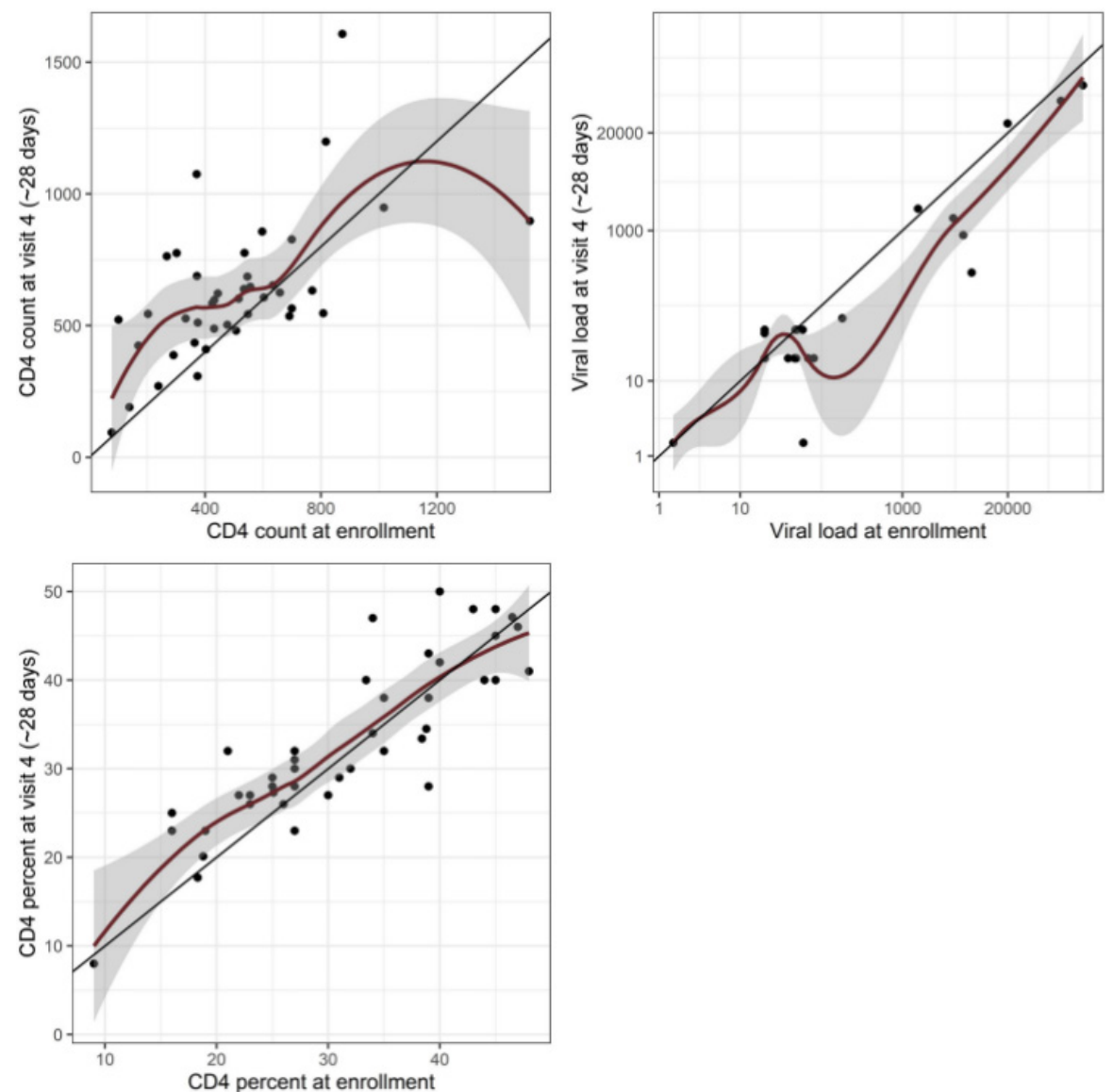

Figure 3 Comparison of enrollment and convalescent CD4 count, \%CD4, and HIV viral load in 42 of the HIV-positive men with ILI. The red lines represent locally weighted scatterplot smoothing, that is, a running average. ILI, influenza-like illness.

in the multiplex assay used in that study, none was detected in the Canadian cohort; however, rhinovirus was the most common virus identified in our study among both HIV-positive and HIV-negative subjects and may have impacted our overall clinical severity scores.

Symptom prevalence and severity were generally similar irrespective of HIV status in our predominantly outpatient cohort of otherwise healthy individuals with ILI, with the exception that HIV-positive subjects reported higher levels of diarrhea and fatigue. There were no appreciable differences in important clinical outcomes, including pneumonia, hospitalization, and need for intensive care between HIV-positive and HIVnegative subjects with ILI. These findings differ from contemporary African studies suggesting increased ILI severity associated with HIV positivity ${ }^{12}$; however, they are comparable to case series data from hospitalized US patients with H1N1pdm09 in which HIVinfected patients had higher rates of diarrhea than non-HIV patients but otherwise similar symptoms and clinical outcomes. ${ }^{9}$ Control of HIV may be a key factor in explaining why HIV status appears to impact ILI severity in LMIC but not HIC cohorts such as ours.

Despite similar symptom severity, more study subjects who were HIV-positive reported receiving antivirals directed at influenza than those who were HIVnegative, suggesting that prescribing practices favor more cautious treatment of ILI in individuals with HIV, even in a setting with open access to care and excellent HIV control. This likely reflects a common perception that HIV increases the risk of complications from influenza. Although a differential time to presentation could theoretically impact receipt of antivirals since antiinfluenza antivirals are typically recommended within 48 hours of symptom onset, the median time to seek care did not differ by HIV status in this study. Receipt of anti-influenza antivirals was not independently associated with symptomatology, nor did antiviral use appear to influence the impact of HIV on composite symptom scores when accounted for in the multivariate analysis. However, we cannot discount the possibility that discrepancy in antiviral use may have impacted viral pathogen recovery or clinical outcomes.

The individuals who comprised the HIV-positive cohort in this study were generally well controlled with intact cellular immunity, as evidenced by low median viral load and median CD4 count of 492 cells $/ \mathrm{mm}^{3}$. The lower absolute CD4 count during acute illness is consistent with previously published results, ${ }^{18}$ as is the lesser impact of acute illness on \%CD4. ${ }^{19}{ }^{20}$ Preserved immune function, as well as a relatively high rate of influenza vaccination (compared with the typical vaccination rate in LMIC) may help account for the low ILI severity in our HIV-positive cohort. ${ }^{21}$ 
This study has several limitations. Restriction of the analysis to adult men to minimize baseline differences between comparator groups limits the applicability of the results to women. Results cannot be extrapolated to a cohort with more comorbidities or poorly controlled HIV; however, this limitation was by design in order to inform on a militarily relevant outpatient population. Also, due to the overall low event rate for important clinical outcomes such as pneumonia and hospitalization, differences may have existed that we did not have adequate power to detect.

In summary, HIV-positive and HIV-negative enrollees in this predominantly outpatient cohort of otherwise healthy adult men with ILI reported a similar illness experience, aside from higher severity of GI symptoms among HIV-positive individuals, particularly those in the younger age range. Whether the higher prevalence of moderate-severe diarrhea reported by subjects with HIV was attributable to the ILI itself, versus due to a combination of factors such as cART, HIV infection, and the ILI pathogen, is not discernible from the available data. There were no apparent differences in clinical outcomes by HIV status, although a higher percentage of subjects with HIV reported receiving antiviral treatment, likely reflecting differences in prescribing practices. Given current national policies promoting increased identification and linkage to care of HIV-infected persons in an enhanced effort to end the HIV epidemic, the otherwise healthy HIV-positive cohort included in this study is likely to be increasingly reflective of the general population of people living with HIV in this country. Thus, in addition to being informative for our military population, these results have potential broader relevance about ILI epidemiology and severity among men living with HIV in the USA.

\author{
Author affiliations \\ ${ }^{1}$ Department of Medicine, Madigan Army Medical Center, Tacoma, Washington, \\ USA \\ ${ }^{2}$ Department of Preventive Medicine and Biostatistics, Uniformed Services \\ University of the Health Sciences, Infectious Disease Clinical Research \\ Program, Bethesda, Maryland, USA \\ ${ }^{3}$ Henry M Jackson Foundation for the Advancement of Military Medicine Inc, \\ Bethesda, Maryland, USA \\ ${ }^{4}$ Department of Medicine, Brooke Army Medical Center, San Antonio, Texas, \\ USA \\ ${ }^{5}$ Infectious Disease Department, Naval Medical Center Portsmouth, \\ Portsmouth, Virginia, USA \\ ${ }^{6}$ Department of Medicine, Naval Medical Center San Diego, San Diego, \\ California, USA \\ ${ }^{7}$ Department of Pediatrics, Naval Medical Center San Diego, San Diego, \\ California, USA \\ ${ }^{8}$ Department of Medicine, Walter Reed National Military Medical Center, \\ Bethesda, Maryland, USA
}

\footnotetext{
Acknowledgements We thank the contributions of the Acute Respiratory Infection Consortium team of clinical research coordinators, clinical site managers, data managers and administrative support personnel to the success of this project.

Contributors All authors provided substantial contributions to the conception or design of the work, or the acquisition, analysis, or interpretation of data for the work. All authors also took part in drafting the work or revising it critically for important intellectual content, provided final approval of the version to be published and agreed to be accountable for all aspects of the work. Each author's specific contributions are further outlined as follows: conception and design of the study/experiments: CS, EVM, CC, and THB;
}

acquisition of data/performance of experiments: CS, REC, SAR, MF, W-JC, PJD, TNL, MR, RCM, JCA, AG, CC, and THB; creation of detailed analysis plan and/ or analysis of the data: CS, REC, CC, EVM, W-JC, and SAR; interpretation of the findings: REC, CS, SAR, RCM, BA, and CC; contribution of resources, reagents/ materials/specimens: BA, EVM, CC, and THB; composition of the first draft of the manuscript: REC, CS, and SAR; provision of critical revisions and edits (scientific content) to provisional drafts and review and approval of the final version for submission: all authors.

Funding This work (Infectious Disease Clinical Research Program (IDCRP)-045) was conducted by the IDCRP, a Department of Defense program executed by the Uniformed Services University of the Health Sciences through a cooperative agreement with The Henry M. Jackson Foundation for the Advancement of Military Medicine, Inc. This project has been funded in whole, or in part, with federal funds from the National Institute of Allergy and Infectious Diseases, National Institutes of Health, under Inter-Agency Agreement Y1-Al-5072, and the Armed Forces Health Surveillance Center, Global Emerging Infections Surveillance and Response System.

Disclaimer The contents of this publication are the sole responsibility of the authors and do not necessarily reflect the views, opinions or policies of the Uniformed Services University of the Health Sciences (USUHS), the Department of Defense (DoD), the Departments of the Army, Navy, or Air Force. Mentions of trade names, commercial products, or organizations do not imply endorsement by the US government. One or more authors are military service members or employees of the US government. This work was prepared as part of their official duties. Title 17 U.S.C. $\$ 105$ provides that 'Copyright protection under this title is not available for any work of the United States Government.' Title 17 U.S.C. §101 defines a US government work as a work prepared by a military service member or employee of the US government as part of that person's official duties. The views expressed are those of the authors and do not reflect the official views of the USUHS, The Henry M. Jackson Foundation for the Advancement of Military Medicine, Inc, the National Institutes of Health or the Department of Health and Human Services, Brooke Army Medical Center, the US Army Medical Department, the US. Army Office of the Surgeon General, the DoD, or the Departments of the Army, Navy or Air Force. The investigators have adhered to the policies for protection of human subjects as prescribed in 45CFR46.

\section{Competing interests None declared.}

Patient consent for publication Not required.

Ethics approval The Infectious Disease Institutional Review Board of the Uniformed Services University approved the study (IDCRP-045).

Provenance and peer review Not commissioned; externally peer reviewed.

Data availability statement Individual subject data will not be available. The study protocol and statistical analysis plan may be available upon reasonable request.

Supplemental material This content has been supplied by the author(s). It has not been vetted by BMJ Publishing Group Limited (BMJ) and may not have been peer-reviewed. Any opinions or recommendations discussed are solely those of the author(s) and are not endorsed by BMJ. BMJ disclaims all liability and responsibility arising from any reliance placed on the content. Where the content includes any translated material, BMJ does not warrant the accuracy and reliability of the translations (including but not limited to local regulations, clinical guidelines, terminology, drug names and drug dosages), and is not responsible for any error and/or omissions arising from translation and adaptation or otherwise.

Open access This is an open access article distributed in accordance with the Creative Commons Attribution Non Commercial (CC BY-NC 4.0) license, which permits others to distribute, remix, adapt, build upon this work noncommercially, and license their derivative works on different terms, provided the original work is properly cited, an indication of whether changes were made, and the use is non-commercial. See: http://creativecommons.org/ licenses/by-nc/4.0/.

\section{ORCID iD}

Rhonda E Colombo http://orcid.org/0000-0003-0154-1928

\section{REFERENCES}

1 Ho A, Aston SJ, Jary H, et al. Impact of human immunodeficiency virus on the burden and severity of influenza illness in Malawian adults: a prospective cohort and parallel case-control study. Clin Infect Dis 2018;66:865-76.

2 Kenmoe S, Bigna JJ, Fatawou Modiyingi A, et al. Case fatality rate and viral aetiologies of acute respiratory tract infections in HIV positive and negative 
people in Africa: the VARIAFRICA-HIV systematic review and meta-analysis. J Clin Virol 2019;117:96-102.

3 Lin JC, Nichol KL. Excess mortality due to pneumonia or influenza during influenza seasons among persons with acquired immunodeficiency syndrome. Arch Intern Med 2001;161:441-6.

4 Neuzil KM, Reed GW, Mitchel EF, et al. Influenza-Associated morbidity and mortality in young and middle-aged women. JAMA 1999;281:901-7.

5 Sheth AN, Althoff KN, Brooks JT. Influenza susceptibility, severity, and shedding in HIV-infected adults: a review of the literature. Clin Infect Dis 2011;52:219-27.

6 Cooper CL. Pandemic H1N12009 influenza and HIV: a review of natural history, management and vaccine immunogenicity. Curr Opin Infect Dis 2012;25:26-35.

7 Patel P, Bush T, Kojic EM, et al. Duration of influenza virus shedding among HIV-infected adults in the CART era, 2010-2011. AIDS Res Hum Retroviruses 2016;32:1180-6.

8 Sheth AN, Patel P, Peters PJ. Influenza and HIV: lessons from the 2009 H1N1 influenza pandemic. Curr HIVIAIDS Rep 2011;8:181-91.

9 Peters PJ, Skarbinski J, Louie JK, et al. HIV-infected hospitalized patients with 2009 pandemic influenza A (pH1N1)--United States, spring and summer 2009. Clin Infect Dis 2011;52 Suppl 1:S183-8.

10 Riera M, Payeras A, Marcos MA, et al. Clinical presentation and prognosis of the 2009 H1N1 influenza A infection in HIV-1-infected patients: a Spanish multicenter study. AIDS 2010;24:2461-7.

11 Martin-Loeches I, Díaz E, Vidaur L, et al. Pandemic and post-pandemic influenza A (H1N1) infection in critically ill patients. Crit Care 2011;15:R286.

12 Coleman BL, Fadel SA, Fitzpatrick T. Risk factors for serious outcomes associated with influenza illness in high-versus low- and middle-income countries: systematic literature review and meta-analysis. Influenza Other Respi Viruses 2018;12:22-9.
13 Coles C, Millar EV, Burgess T, et al. The acute respiratory infection Consortium: a multi-site, multi-disciplinary clinical research network in the Department of defense. Mil Med 2019;184:44-50.

14 Schofield C, Colombo RE, Richard SA, et al. Comparable disease severity by influenza virus subtype in the acute respiratory infection Consortium natural history study. Mil Med 2020;185:e1008-15.

15 R Core Team. R: a language and environment for statistical computing. Vienna, Austria: R Foundation for Statistical Computing, 2018. https:// www.R-project.org/

16 Pinheiro J, Bates D, DebRoy S, et al. R core team (2018)._nlme: linear and nonlinear mixed effects models. R package version 3.1-137. Available: https:// CRAN.Rproject.org/package $=$ nlme

17 Klein MB, Yang H, DelBalso L, et al. Viral pathogens including human metapneumovirus are the primary cause of febrile respiratory illness in HIV-infected adults receiving antiretroviral therapy. J Infect Dis 2010;201:297-301.

18 Feeney C, Bryzman S, Kong L, et al. T-lymphocyte subsets in acute illness. Crit Care Med 1995:23:1680-5.

19 Fantin B, Joly V, Elbim C, et al. Lymphocyte subset counts during the course of community-acquired pneumonia: evolution according to age, human immunodeficiency virus status, and etiologic microorganisms. Clin Infect Dis 1996;22:1096-8.

20 Panel on Antiretroviral Guidelines for Adults and Adolescents. Guidelines for the use of antiretroviral agents in adults and adolescents with HIV. Department of health and human services. Available: http://www.aidsinfo.nih.gov/ ContentFiles/AdultandAdolescentGL.pdf [Accessed 1 Mar 2020].

21 Ortiz JR, Neuzil KM. Influenza immunization in low- and middle-income countries: preparing for next-generation influenza vaccines. J Infect Dis 2019:219:\$97-106. 